\title{
A Design and Implement of Portable Epidemic Detection Device based on STM32
}

\author{
Yuqi Zhao, Yizhun Peng*, Xiaowei Wu, Yusong Zhou \\ College of Electronic Information and Automation, \\ Tianjin University of Science and Technology, Tianjin, 300222, China; \\ E-mail:*pengyizhun@tust.edu.cn \\ www.tust.edu.cn
}

\begin{abstract}
The equipment is mainly designed for epidemic detection. It is an intelligent detection box device. It collects and analyzes data through cameras and sensors. It uses LBP algorithm and LAB color model to enable the detection box to identify the temperature and mask of the entered personnel. Wearing conditions, preliminary comparison of epidemic prevention standards for passers-by, whether to wear masks, whether the body temperature exceeds the standard, and at the same time enter the identity of new personnel and store them in the database, and detect the incoming personnel through face recognition to find out in time Those who are not entered shall take corresponding measures in time to improve the efficiency of epidemic prevention and control. The device does not need to be held in hand, can be fixed anywhere, can effectively avoid cross-infection, and supports self-set temperature alarm thresholds, support voice broadcast reminders, fast and accurate non-contact temperature measurement.
\end{abstract}

Keywords: Non-contact temperature measurement, face recognition, mask recognition, identity collection

\section{Introduction}

Since the first case of unidentified pneumonia appeared in Wuhan on December 8, 2019, to December 1, 2020, a total of 93,889 new coronary pneumonia patients have been diagnosed nationwide, of which 87,707 cases have been effectively cured, accounting for approximately the total number of confirmed cases Of $93.41 \%$.

Since the new crown epidemic, domestic measures have been effectively implemented ${ }^{1}$. A large number of detection equipment has been used in epidemic prevention and control, and there has been a relatively large market in China and the world. At the same time, the functional requirements of detection equipment have become higher and higher. Non-contact body temperature detection, mask wearing detection, identity entry, etc. can achieve precise identification and detection. The accuracy of the infrared thermometer is related to four key factors: sensor, ADC accuracy, algorithm, and the structure and production process of the forehead thermometer. So we designed a smart detection box to make detection more convenient and quicker and improve the efficiency of prevention and control.

\section{System overview}

The device is a temperature detection box with identification. The detection box can realize non-contact temperature recognition, face recognition, identity comparison, mask recognition, voice prompts and other functions, data analysis through ${ }^{2}$ camera and sensor recognition, and human-computer interaction through OLED display. The main functions that can be achieved are: 1. Non-contact temperature detection, and at the same time, by setting the alarm threshold, a faster and more accurate identification of epidemic prevention standards can be achieved, and then voice broadcast reminders can effectively improve the prevention and control efficiency. 2. Data collection through the camera, real-time data transmission through the serial port, data

(C) The 2021 International Conference on Artificial Life and Robotics (ICAROB2021), January 21 to 24, 2021 
analysis through the MCU, and then voice broadcast of the mask wearing situation, mainly used for standard mask recognition in the current epidemic. 3. Through oled display, perform human-computer interaction, enter face data, and store it in the database, so that face information can be effectively recognized, identity recognition, and prevention and control become more efficient. 4. The device does not need to be held in hand and can be fixed anywhere, which can effectively avoid cross-infection.It also supports self-settable temperature alarm thresholds, voice broadcast reminders, and fast and accurate non-contact temperature measurement. 。

\section{The hardware structure design}

\subsection{Core controller}

The core controller of the device uses the stm $32 \mathrm{f} 1$ series chip, which has obvious characteristics. ARM's high-performance "Cortex-M3" core: 1.25DMips/MHz, while the ARM7TDMI is only 0.95DMips/MHz, first-class peripherals. $1 \mu \mathrm{s}$ dual 12-bit ADC, 4Mbit/s UART, $18 \mathrm{Mbit} / \mathrm{s} \mathrm{SPI}, 18 \mathrm{MHz}$ I/O flip speed. Low power consumption, consumes $36 \mathrm{~mA}$ at $72 \mathrm{MHz}$ (all peripherals are in working state), drops to $2 \mu \mathrm{A}$ in standby, maximum integration, reset circuit, low voltage detection, voltage regulator, precise RC oscillator, etc ${ }^{3}$.

\subsection{Image acquisition equipment}

The device uses an openmv4 camera, which is an open source, low-cost, and powerful machine vision module. With STM32F427CPU as the core, it integrates the OV7725 camera chip. On the small hardware module, the core machine vision algorithm is efficiently implemented in $\mathrm{C}$ language, and the Python programming interface is provided. The adopted STM32F427 has abundant hardware resources, leading to UART, I2C, SPI, PWM, ADC, DAC and GPIO interfaces to facilitate the expansion of peripheral functions. The USB interface is used to connect to the integrated development environment OpenMVIDE on the computer to assist in programming, debugging and updating firmware. The TF card slot supports large-capacity TF cards, which can be used to store programs and save photos. The open source environment and rich Python libraries make it very conducive to the development of visual identity.

\subsection{Sensor}

The MLX90614 infrared temperature sensor is used. MLX90614 is an infrared thermometer for non-contact temperature measurement. Infrared temperature measurement is to determine the temperature of the object according to the infrared radiation energy of the object to be measured. It does not contact the object to be measured, and has no influence on the temperature distribution field of the object to be measured. It has high temperature resolution, fast response speed, and wide temperature measurement range. Restricted by the upper limit of temperature measurement, good stability and other characteristics. The measured target temperature and ambient temperature of MLX90614 are output through the IIC interface. The sensor is directly connected to the core board, and is packaged as a temperature measurement structure through a printed external package. At the same time, the circuit is connected in series with an infrared indicator light to confirm and indicate the object under test.

We need to read the data from the MLX90614 infrared temperature sensor, no matter whether the data is in EEPROM or RAM, we can read it in the same way. We have already said that the command byte for operating 


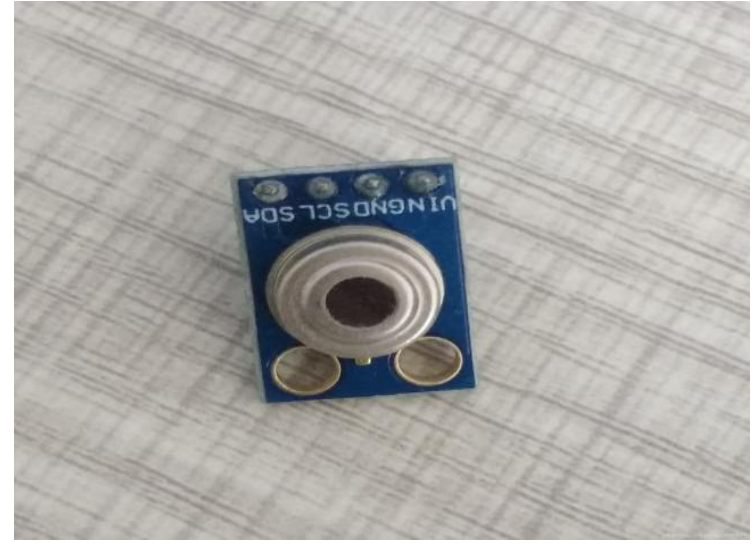

Fig.1 MLX90614 infrared temperature sensor

EEPROM and RAM consists of a 3-bit command and a 5-bit address. We implement the data reading function as follows:

\begin{tabular}{|c|c|c|c|c|c|c|c|c|c|c|c|}
\hline 1 & & 7 & 1 & 1 & & 8 & 1 & 1 & 7 & 1 & 1 \\
\hline \multirow[t]{3}{*}{$s$} & & Address & $\mathrm{wr}$ & A & & Command & A & $\mathrm{Sr}$ & Slave Address & $\mathrm{Rd}$ & A \\
\hline & & & & & 1 & 8 & & 1 & 8 & 1 & 1 \\
\hline & & Data & Low & & A & Data Byte Higł & & A & PEC & A & P \\
\hline
\end{tabular}

Fig.2 Data reading function

\subsection{Display}

The display uses an OLED display module, OLED, or Organic Light-Emitting Diode (Organic Light-Emitting Diode), also known as Organic Electroluminesence Display (OELD). OLED has excellent characteristics such as self-luminous, no backlight, high contrast, thin thickness, wide viewing angle, fast response speed, can be used for flexible panels, wide operating temperature range, simple structure and manufacturing process, etc. It is considered to be The next-generation flat panel display emerging application technology.

OLED display technology has self-luminous characteristics. It uses very thin organic material coatings and glass substrates. When current passes through, these organic materials will emit light. The OLED display screen has a large viewing angle and can save power. This kind of display device has been used in MP3 players since 1988.

LCDs require backlighting, but OLEDs do not, because it is self-luminous. For the same display, the OLED effect should be better. With current technology, it is difficult to increase the size of OLEDs, but the resolution can be very high.

The oled display is as follows, which can effectively carry out human-computer interaction

\subsection{Voice broadcast}

The voice broadcast function of the device is realized by syn6288 module, which broadcasts through the speaker. The SYN6288 Chinese speech synthesis chip used by Speech Synthesizer Bee is a speech synthesis chip with high cost performance, more natural speech synthesis, and for high-end applications ${ }^{4}$. SYN6288 receives the text to be synthesized through the asynchronous serial port, and realizes the conversion of text to voice (TTS). The module is directly connected to the MCU.

\section{System process}

\subsection{System operation process}

According to the needs of users, the detection mode is selected. The detection mode includes mask recognition, identity entry and temperature detection.

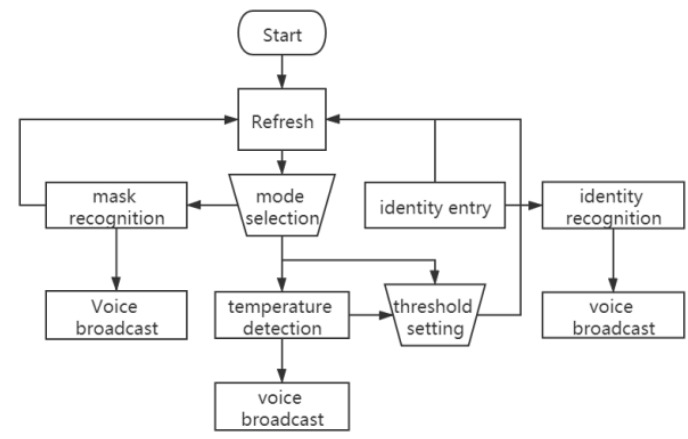

Fig.3 System operation process

When the user enters the mask recognition, the camera module will automatically turn on to take pictures, compare and analyze the photographed image with the mask recognition set that has been trained, and get the result. Mask recognition is based on the neural network 
model trained by openmv using EDGE IMPULSE's online website. Training the neural network model is to perform machine learning. Through machine learning, you can determine whether there are people in the photo and whether to wear masks and other functions. By establishing two categories, one face and one mask, one hundred images are collected each, and the collected data is sent to the EDGE IMPULSE website for model training. After DSP preprocessing, the following analysis is obtained:

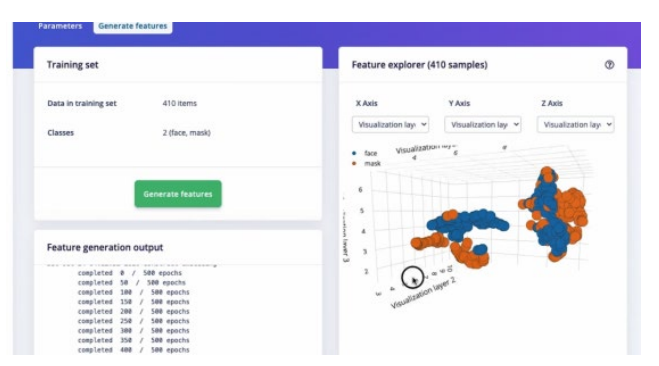

Fig.4 DSP preprocessing results

It can be seen that the two colors of the training model face and mask are clearly distinguished and are not mixed together. After ten rounds of training, the training data obtained is as follows:

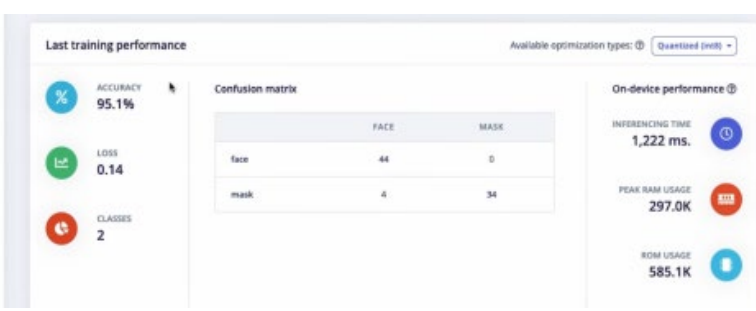

Fig.5 DSP training data

It can be seen that our mask recognition model has an accuracy of $95.1 \%$. Run the training set on openmv and the mask recognition is completed.

\subsection{Detection processing}

After the user enters the temperature detection, the threshold value of the temperature measurement device can be set by key operation ${ }^{5}$, so that the high temperature alarm system remains effective. After the MLX90614 non-contact temperature measurement module collects
(DataH:DataL) data, according to the formula:

\section{$\mathrm{T}=($ DataH:DataL $) * 0.02-273.15$}

To get the final temperature $\mathrm{T}$, we use the bubble sorting method when collecting, and each temperature measurement will collect ten times of data. After sorting, remove the abnormal temperature value and average it as the final temperature measurement data, so as to avoid abnormal temperature And make the final temperature data more accurate.

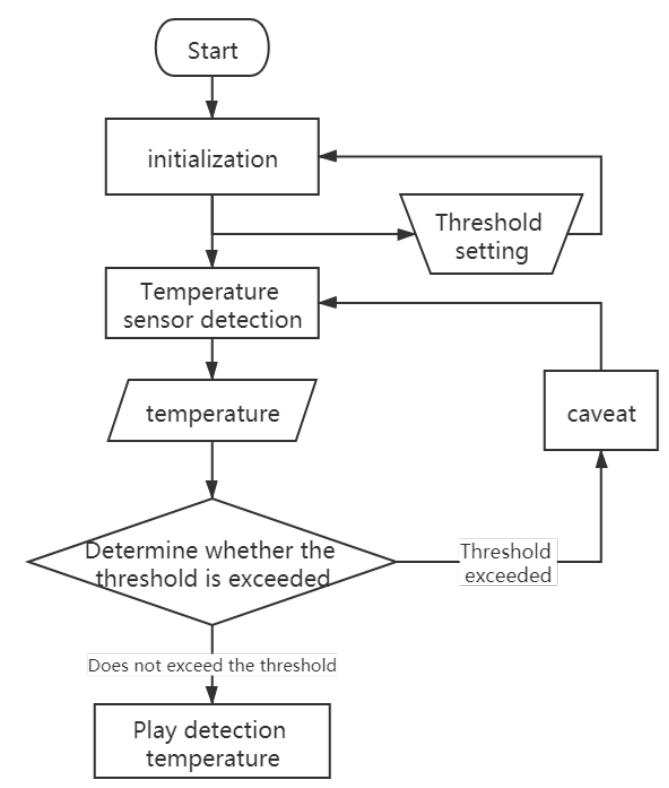

Fig.6 Temperature measurement flow chart

(C) The 2021 International Conference on Artificial Life and Robotics (ICAROB2021), January 21 to 24, 2021 

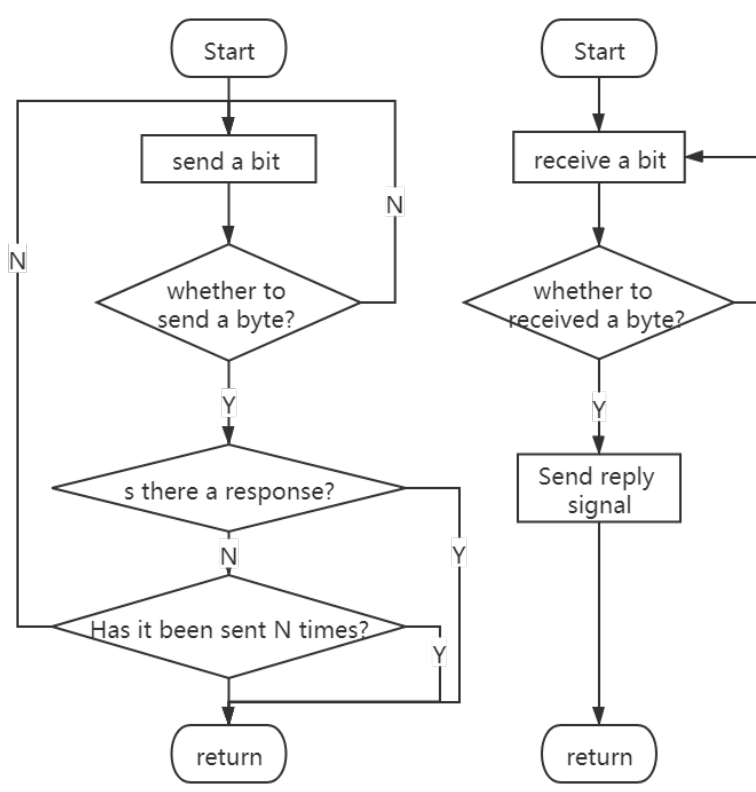

Fig.7 MLX90614 send and receive byte flow chart

\subsection{System circuit design}

The system is powered by $5 \mathrm{v}$ with energy, voltage conversion is connected to the core board through a voltage stabilizing module, the circuit board adopts a homemade pcb circuit board, integrated oled, MLX90614, openmv and power interface, which can make the signal transmission more stable and reduce external signal interference, To ensure the stability and accuracy of the test results.

\section{Test and conclusion}

\subsection{Test plan}

- The encapsulated temperature detection gun is used for non-contact detection of different water temperatures, such as $35.7^{\circ} \mathrm{C}, 41.2^{\circ} \mathrm{C}$, and $80^{\circ} \mathrm{C}$, and the results are tested with the threshold $25-48^{\circ} \mathrm{C}$ set in advance

- Measure human body temperature, floor temperature, desktop temperature without contact within a certain distance
- Enter the identity in advance, save it in the database, and then perform face recognition and check the face recognition function

- There are two cases of wearing masks and not wearing masks by three people, and testing the three groups wearing masks to test their stability

- Detect the on-site deep learning function of the device, Provide three unfamiliar faces, learn their facial identity within 5 minutes and record them in the database, and then the temperature measuring box identifies whether they are the input personnel, and detects whether they have the function of deep learning

\section{2. $\quad$ Test results}

After repeated testing, the device has the above functions, and can identify and detect very stably. It can quickly and accurately identify human body temperature and water temperature and broadcast it. It can accurately and stably recognize the wearing of a mask. It can learn on-site, automatically enter the identity and recognize it. . The temperature detection efficiency is greatly improved, and the identification is accurately prevented and controlled.

\section{References}

1. Yang Li, Yang Zhen "Principle and Technology of Infrared Thermal Imaging Temperature Measurement" Science Press, 2012.6.

2. Li Min, "Design of Wireless Infrared Temperature Measurement System" Guangdong Vocational College of Agriculture, Industry and Commerce, 2020.3.

3. Zheng Zaisheng "Research on Automatic Fare Collection System Based on Face Recognition" China Computer Room Facilities Engineering Co., Ltd., 2020.9

4. Li Chengyong, Tan Hanzhong, Wang Sha, et al. Intelligent "seeking" car control system based on Open MV[J]. Liquid Crystal Display,2020,08:870-876.

5. Ye Siyan, Zhang Yongning, Li Jin, et al. Design of smart follow luggage based on UWB positioning and Open MV recognition[J]. Journal of Chengdu University of Technology,2020,03:39-33. 